\title{
Maternal morbidity of adolescent pregnant women
}

\section{${ }^{1}$ Carlos Arturo Paniagua Coahuila, ${ }^{1}$ José Anaya Herrera, ${ }^{2}$ Dulce Alejandra Alonso Lozano, ${ }^{1}$ Lenny Piña Guerrero, ${ }^{3}$ Raúl Alejandro Miranda Ojeda, ${ }^{\star 1,3}$ Hugo Mendieta Zerón}

${ }^{1}$ Obstetrics and Gynecology Department, Mónica Pretelini Sáenz Maternal-Perinatal Hospital, Toluca, Mexico

${ }^{2}$ Gynecology and Obstetrics Hospital, Mother-Child Institute of the State of Mexico, Toluca, Mexico

${ }^{3}$ Research Unit, Faculty of Medicine, Autonomous University of the State of Mexico, Toluca, Mexico

Authors' ORCID iDs, academic degrees and contributions are available at the end of the article

${ }^{\star}$ Corresponding author - Hugo Mendieta Zerón, e-mail: drmendietaz@yahoo.com

Manuscript received October 18, 2021; revised manuscript December 03, 2021; published online December 17, 2021

\begin{abstract}
Background: In Mexico, it is estimated that the adolescent population represents $29 \%$ of the population of childbearing age. The present study aimed to analyze the obstetric results of 3310 adolescent pregnant women attended in a third level hospital.

Material and methods: All records of pregnant women aged 19 years or less up to the date of admission were analyzed at the Mónica Pretelini Saenz Maternal Perinatal Hospital during the period from January 2018 to June 2020, with the following variables: age, pregnancy, resolution obstetric, severe preeclampsia, preeclampsia and gestational hypertension.

Results: A total of 13874 pregnant women were attended, of which 3310 (24\%) patients were adolescents. The overall frequency of obstetric complications was $21 \%$, including obstetric hemorrhage (13\%) and hypertensive disorders of pregnancy ( $8 \%)$. Regarding postpartum obstetric hemorrhage events, classified according to the Advanced Trauma Life Support shock scale, they were categorized as Grade 1 - 338 cases, Grade II - 76 cases, Grade III - 11 cases and Grade IV - 1 case. Hypertensive disorders of pregnancy highlight preeclampsia as the most frequent with a total of 97 cases, followed by 89 cases of severe preeclampsia, 58 cases of gestational hypertension, 14 cases of chronic hypertension and 3 cases of chronic hypertension with preeclampsia. Conclusions: The main complications found in the Mexican pregnant adolescent population were obstetric hemorrhage, which was more frequent in the population aged 15 to 19 years, and hypertensive disorders, which occurred more frequently in the population aged 9 to 14 years.

Key words: adolescence, maternal morbidity, preeclampsia.
\end{abstract}

\section{Cite this article}

Paniagua Coahuila CA, Anaya Herrera J, Alonso Lozano DA, Piña Guerrero L, Miranda Ojeda RA, Mendieta Zerón H. Maternal morbidity of adolescent pregnant women. Mold Med J. 2021;64(6):10-12. https://doi.org/10.52418/moldovan-med-j.64-6.21.02.

\section{Introduction}

According to the World Health Organization (WHO), an average of 16 million adolescents, between 15 and 19 years of age, account for $11 \%$ of annual births worldwide. The WHO defines adolescence as the period of human development between childhood and adulthood (early adolescence: $10-14$ years and late adolescence: $15-19$ years) [1].

In Mexico, it is estimated that the adolescent population represents just over $18 \%$ of the total population and $29 \%$ of the population of childbearing age; the main biological problem of adolescent pregnancy is the lack of maturity for gestation. A determining factor of reproductive health in adolescents is reflected in the first trimester, when there is the importance of transgenerational events that make it possible to reduce the main diseases that affect pregnancy, such as diabetes, hypertension, cancer, preeclampsia, prematurity, intrauterine growth restriction, etc. [2].

Complications during pregnancy and childbirth are the second leading cause of mortality worldwide in this population. In this regard, the WHO reports that 830 women die daily from these complications and that $95 \%$ of them occur in developing countries [3]; in Latin America, almost half of the female population has had at least one delivery before the age of 20 years [4].

In the same line, the National Institute of Statistics, Geography and Informatics (INEGI), in 2018 published the data informing that in Mexico $17.5 \%$ of births are to teenage mothers; and teenage pregnancy continues to be one of the main contributors to maternal and infant mortality. Complications of pregnancy and childbirth are the leading cause of death among girls aged 10-19 years worldwide [5, 6].

Adolescent mothers (aged 10-19 years) face higher risks of eclampsia, puerperal endometritis, and systemic infections than women aged 20-24 years [4]. Besides, about 3.9 million unsafe abortions occur among girls aged 13 to 19 years each year, contributing to maternal mortality and long-lasting health problems [7]. Also, the emotional, psychological, and social needs of pregnant adolescents may be greater than those of other women.

Early childbearing can increase risks for newborns as well as for young mothers. In low- and middle-income 
countries, babies born to mothers younger than 19 years face increased risks of low birth weight, preterm delivery, and serious neonatal conditions [4]. In some settings, rapid repeated pregnancy is common for young mothers, presenting additional risks to both mother and child [8].

Some research has concluded that these adverse events are due to age, and even to biological immaturity that may predispose very young mothers to subclinical infections; to an increase in the production of prostaglandins, with a consequent increase in the incidence of preterm delivery and fetal growth restriction $[9,10]$. The present study had the purpose of analyzing the obstetric outcomes of adolescent pregnant women attended in a third level hospital in Toluca, Mexico.

\section{Material and methods}

This was a retrospective and descriptive study of patients admitted to the Mónica Pretelini Sáenz Maternal Perinatal Hospital (MPSMPH), Health Institute of the State of Mexico (HISM), Toluca, Mexico.

All the records of pregnant women aged 19 years from January 2018 to June 2020 were analyzed with the following variables: age, gestation, obstetric resolution, severe preeclampsia, preeclampsia, and gestational hypertension.

The present study followed the ethical principles of human research stipulated in the Declaration of Helsinki [11] and was considered risk-free since only information from the hospital databases was collected. The ethics committee MPSMPH, approved the protocol (code: 2021-03-721).

The descriptive analysis was performed according to the nature of the variables: continuous variables were expressed as means and medians with standard deviation, while categorical variables were summarized as frequencies and percentages. Also, a measur were used for continuous variables. All statistical analyses were performed using the SPS ${ }^{\circledR}$ program, version 23.0.

\section{Results}

During the time of this survey, 13874 pregnant women were attended in the MPSMPH, of which 3310 patients were adolescents $(24 \%)$. The median age was 17 years (range 9 to 19 years), $42.5 \%$ of the patients were between 17 and 18 years old, however, 908 patients were younger than 15 years old and there was a record of 4 patients aged 9 years. 59\% (1952) resolved the pregnancy vaginally, 33\% (1081) through the abdominal route, and $8 \%$ (277) underwent uterine evacuation.

The overall frequency of obstetric complications was $21 \%$, including obstetric hemorrhage (13\%) and hypertensive disorders of pregnancy $(8 \%)$; but there were no maternal deaths. The diagnosis of hypertensive disorders of pregnancy was made according to the time of onset of clinical data, gestational age of onset, and biochemical alterations. The most frequent diagnosis was preeclampsia with a total of 97 cases, followed by preeclampsia with a severity of 89 cases, gestational hypertension - 58 cases, chronic hypertension with 14 cases, and chronic hypertension with added preeclampsia with 3 cases. It should be noted that there were no cases of eclampsia, as well as admissions to the Obstetric Intensive Care Unit (O-ICU) with a total of 256 cases.

As for postpartum obstetric hemorrhage events, classified according to the Advanced Trauma Life Support shock scale, they were categorized only according to the estimated amount of loss: Grade I with a total of 338 cases, Grade II with 76 cases, Grade III with 11 cases, and Grade IV with only 1 case that was sent to the O-ICU.

\section{Discussion}

Compared to other Latin American countries, the timing of sexual debut in Mexico is later [12]. However, 15\% of our population were between 9 and 16 years old; thus, it can be affirmed that there are indicative data that the sexual life beginning of the Mexican population is moving to early ages. The results of this study may be useful, for example, to estimate at what age it is convenient to vaccinate young people against human papillomavirus or to know when it is the right time to include information on family planning methods.

It was documented that the resolution of pregnancy was 59\% vaginally, which is consistent with the findings of Amaya J. [9] and Fory JL. [10], where 33\% of pregnancies were resolved by cesarean section; disagreeing with a study conducted in Nepal by Maharjan M. et al. [13], where they studied 2050 pregnant adolescents and reported a cesarean section rate of $11.9 \%$, well below our results; the variation could be because this study was conducted in a rural hospital. According to the Nepal Demographic Health Survey in 2016, the total cesarean section in adolescents constituted 24\%; adolescent pregnancy is higher in South Asian countries due to the common practice of early marriage and the social expectation of having a child soon after marriage.

Garcia-Salgado A. et al. in 2019 [14] mentioned that adolescent pregnancy carries a five times higher risk of maternal mortality compared to adult pregnant women; however, at the MPSMPH adolescent maternal deaths in the period from January 2018 to June 2020 were not confirmed; attributing it to the interdisciplinary management of this unit. The results of the present study show that adolescent pregnancy is associated with complications due mainly to obstetric hemorrhage and hypertensive disorders of pregnancy, these findings are consistent with those reported in the literature consulted [10, 14-18].

Finally, eight percent of the pregnant adolescents presented hypertensive diseases; most frequently preeclampsia (97) and preeclampsia with severity (89); studies show that adolescents of younger ages ( $<15$ years) seem to be the most prone to suffer from this complication $[14,17]$.

Although no statistically significant differences were observed in the present study concerning the frequency of threatened abortion, urinary tract infections, gestational diabetes, and ectopic pregnancies, a higher frequency of obstetric hemorrhage episodes were detected, representing $13 \%$ of pregnant adolescents, in agreement with studies conducted in populations, such as Argentina, Guatemala, India, Kenya, Pakistan and Zambia [17] and international, multi- 
center registries from countries with limited resources [19].

\section{Conclusions}

The main complications encountered in Mexican pregnant adolescent population were obstetric hemorrhage, which was more frequent in the population aged 15-19 years and hypertensive disorders, which occurred more frequently in the population aged 9-14 years.

Current health programs have privileged the care of children and the elderly, leaving the adolescent population uncovered.

\section{References}

1. World Health Organization. Adolescent pregnancy [Internet]. Geneva: WHO; 2020 [cited 2021 Oct 13]. Available from: https://www.who.int/ news-room/fact-sheets/detail/adolescent-pregnancy

2. Deichmann U. Epigenetics: the origins and evolution of a fashionable topic. Dev Biol. 2016;416(1):249-54. doi: 10.1016/j.ydbio.2016.06.005.

3. World Health Organization. World health statistics 2013 [Internet]. Geneva: WHO; 2013 [cited 2021 Oct 13]. Available from: https://www.who.int/gho/ publications/world_health_statistics/EN_WHS2013_Full.pdf

4. Ganchimeg T, Ota E, Morisaki N, Laopaiboon M, Lumbiganon P, Zhang J, et al. Pregnancy and childbirth outcomes among adolescent mothers: a World Health Organization multicountry study. BJOG. 2014;121 Suppl 1:40-8. doi: 10.1111/1471-0528.12630.

5. Instituto Nacional de Estadística y Geografía (INEGI) [National Institute of Statistics and Geography]. Natalidad y fecundidad [Natality and fertility] [Internet]. Aguascalientes: INEGI; 2021 [cited 2021 Feb 13]. Available from: https://www.inegi.org.mx/temas/natalidad/. Spanish.

6. World Health Organization, et al. Trends in maternal mortality: 1990 to 2015: estimates by WHO, UNICEF, UNFPA, World Bank Group and the United Nations Population Division [Internet]. Geneva: WHO; 2015 [cited 2021 Sep 13]. Available from: http://www.who.int/reproductivehealth/ publications/monitoring/maternal-mortality-2015/en/

7. Darroch JE, Woog V, Bankole A, Ashford LS. Adding it Up: costs and benefits of meeting the contraceptive needs of adolescents. New York: Guttmacher Institute; 2016 [cited 2021 Oct 18]. Available from: https:// www.guttmacher.org/report/adding-it-meeting-contraceptive-needs-ofadolescents

8. Kozuki N, Lee AC, Silveira MF, Victora CG, Adair L, Humphrey J, et al. The associations of birth intervals with small-for-gestational-age, preterm, and neonatal and infant mortality: a meta-analysis. BMC Public Health. 2013;13(Suppl 3):S3. doi: 10.1186/1471-2458-13-S3-S3.
9. Amaya J, Borrero C, Ucrós S. Estudio analítico del resultado del embarazo en adolescentes y mujeres de 20 a 29 años en Bogotá [Analytical study of the pregnancy outcome comparing adolescent women and women between 20 to 29 years of age in Bogotá (Colombia)]. Rev Colomb Obstet Ginecol. 2005;56(3):216-24. doi: https://doi.org/10.18597/rcog.527. Spanish.

10. Fory JA, Olivera MJ. Characterization of pregnant adolescents treated between 2012 and 2015 at Central Military Hospital in Bogotá D.C., Colombia Rev Fac Med. 2020;68(2):202-6. doi: 10.15446/revfacmed.v68n2.70818.

11. World Medical Association. WMA Declaration of Helsinki - Ethical Principles for Medical Research Involving Human Subjects [Internet]. Ferney-Voltaire: WMA; 2018 [cited 2021 October 18]. Available from: https://www.wma.net/policies-post/wma-declaration-of-helsinki-ethicalprinciples-for-medical-research-involving-human-subjects/

12. Gálvez-Buccollini JA, DeLea S, Herrera PM, Gilman RH, Paz-Soldan V. Sexual behavior and drug consumption among young adults in a shantytown in Lima, Peru. BMC Public Health. 2009;9:23. doi: 10.1186/14712458-9-23.

13. Maharjan M, Thapa N, Maharjan N, Rai P, Pun P, Petrini MA, et al. Prevalence of teenage pregnancy in a community hospital of rural Nepal: a cross-sectional study. J Nepal Med Assoc. 2019;57(217):176-180. doi: 10.31729/jnma.4083.

14. García-Salgado A, Sánchez-Chávez S, González-Aldeco PM. Embarazo adolescente: resultados obstétricos [Teenage pregnancy: obstetric results]. Rev Hosp Juárez Mexico. 2017;84(1):8-14. Spanish.

15. Watcharaseranee N, Pinchantra P, Piyaman S. The incidence and complications of teenage pregnancy at Chonburi Hospital. J Med Assoc Thai. 2006;89 Suppl 4:S118-123.

16. Chen XK, Wen SW, Fleming N, Demissie K, Rhoads GG, Walker M. Teenage pregnancy and adverse birth outcomes: a large population-based retrospective cohort study. Int J Epidemiol. 2007;36(2):368-73. doi: 10.1093/ ije/dyl284.

17. Althabe F, Moore JL, Gibbons L, Berrueta M, Goudar SS, Chomba E, et al. Adverse maternal and perinatal outcomes in adolescent pregnancies: The Global Network's Maternal Newborn Health Registry study. Reprod Health. 2015;12 Suppl 2(Suppl 2):S8. doi: 10.1186/1742-4755-12-S2-S8.

18. Minjares-Granillo RO, Reza-López SA, Caballero-Valdez S, LevarioCarrillo M, Chávez-Corral DV. Maternal and perinatal outcomes among adolescents and mature women: a hospital-based study in the north of Mexico. J Pediatr Adolesc Gynecol. 2016;29(3):304-11. doi: 10.1016/j. jpag.2015.11.005.

19. Bose CL, Bauserman M, Goldenberg RL, Goudar SS, McClure EM, Pasha O, et al. The Global Network Maternal Newborn Health Registry: a multi-national, community-based registry of pregnancy outcomes. Reprod Health. 2015;12 Suppl 2(Suppl 2):S1. doi: 10.1186/1742-4755-12-S2-S1.

\author{
Authors' ORCID iDs and academic degrees \\ Carlos Arturo Paniagua Coahuila, MD, PhD, Professor - https://orcid.org/0000-0002-4551-0923 \\ José Anaya Herrera, MD - https://orcid.org/0000-0002-1804-1017 \\ Dulce Alejandra Alonso Lozano, MD - https://orcid.org/0000-0003-2023-0498 \\ Lenny Piña Guerrero, PsychoD - https://orcid.org/0000-0003-4264-9089 \\ Raúl Alejandro Miranda Ojeda, MD Student, Research Assistant - https://orcid.org/0000-0003-1896-9648 \\ Hugo Mendieta Zerón, MD, PhD, Professor - https://orcid.org/0000-0003-3492-8950
}

\title{
Authors' contribution
}

CAPC conceptualized the project and designed the research, collected the data and drafted the manuscript; JAH interpreted the data and drafted the manuscript; DAAL interpreted the data, drafted the manuscript and revised the manuscript critically; LPG collected the data; RAMO revised the manuscript critically; HMZ made statistical analysis, drafted the manuscript and revised the manuscript critically. All the authors revised and approved the final version of the manuscript.

Funding

Self-funding.

Ethics approval and consent to participate

This study was approved by the Research Ethics Committee of Mónica Pretelini Sáenz Maternal-Perinatal Hospital, Toluca, Mexico (the protocol code: 2021-03-721).

\section{Conflict of interests}

No competing interests were disclosed. 